Article

\title{
Impact of Right Atrial Physiology on Heart Failure and Adverse Events after Myocardial Infarction
}

\author{
Andreas Schuster 1,2,*,+(D), Sören J. Backhaus ${ }^{1,+}{ }^{\text {, Thomas Stiermaier }}{ }^{3}$, Jenny-Lou Navarra ${ }^{1}$, \\ Johannes Uhlig ${ }^{4} \mathbb{D}^{D}$, Karl-Philipp Rommel ${ }^{5} \mathbb{D}$, Alexander Koschalka ${ }^{1}$, Johannes T. Kowallick ${ }^{4}$, \\ Boris Bigalke ${ }^{6}$, Shelby Kutty ${ }^{7}$, Matthias Gutberlet ${ }^{8}$, Gerd Hasenfuß ${ }^{1}$, Holger Thiele ${ }^{5,+}$ and \\ Ingo Eitel ${ }^{3,+}$
}

1 Department of Cardiology and Pneumology, University Medical Center Göttingen, Georg-August University, German Center for Cardiovascular Research (DZHK), 37075 Göttingen, Germany; soeren.backhaus@outlook.de (S.J.B.); jenny-lou.navarra@gmx.de (J.-L.N.); alex.koscha@gmx.de (A.K.); hasenfus@med.uni-goettingen.de (G.H.)

2 Department of Cardiology, Royal North Shore Hospital, The Kolling Institute, Northern Clinical School, University of Sydney, Sydney 2065, Australia

3 University Heart Center Lübeck, Medical Clinic II (Cardiology/Angiology/Intensive Care Medicine), University Hospital Schleswig-Holstein, German Center for Cardiovascular Research (DZHK), 23538 Lübeck, Germany; Thomas.Stiermaier@uksh.de (T.S.); ingoeitel@gmx.de (I.E.)

4 University Medical Center Göttingen, Institute for Diagnostic and Interventional Radiology, Georg-August University, German Center for Cardiovascular Research (DZHK), 37075 Göttingen, Germany; johannes.uhlig@med.uni-goettingen.de (J.U.); johannes.kowallick@med.uni-goettingen.de (J.T.K.)

5 Department of Internal Medicine/Cardiology, Heart Center Leipzig at University of Leipzig, Leipzig Heart Institute, 04289 Leipzig, Germany; Karl_Ph_Rommel@web.de (K.-P.R.); Holger.Thiele@medizin.uni-leipzig.de (H.T.)

6 Department of Cardiology and Pneumology, Charité Campus Benjamin Franklin, University Medical Center Berlin, 12203 Berlin, Germany; dr.bigalke@gmx.de

7 Taussig Heart Center, Johns Hopkins Hospital, Baltimore, MD 21287, USA; skutty1@jhmi.edu

8 Department of Radiology, Heart Center Leipzig, University of Leipzig, 04289 Leipzig, Germany; Matthias.Gutberlet@medizin.uni-leipzig.de

* Correspondence: andreas_schuster@gmx.net; Tel.: +49-551-39-20870; Fax: +49-551-39-22026

+ Equal contribution as first and senior authors.

Received: 9 December 2019; Accepted: 6 January 2020; Published: 12 January 2020

\begin{abstract}
Background: Right ventricular (RV) function is a known predictor of adverse events in heart failure and following acute myocardial infarction (AMI). While right atrial (RA) involvement is well characterized in pulmonary arterial hypertension, its relative contributions to adverse events following AMI especially in patients with heart failure and congestion need further evaluation. Methods: In this cardiovascular magnetic resonance (CMR)-substudy of AIDA STEMI and TATORT NSTEMI, 1235 AMI patients underwent CMR after primary percutaneous coronary intervention (PCI) in 15 centers across Germany ( $n=795$ with ST-elevation myocardial infarction and 440 with non-ST-elevation MI). Right atrial (RA) performance was evaluated using CMR myocardial feature tracking (CMR-FT) for the assessment of RA reservoir (total strain $\varepsilon_{\mathrm{s}}$ ), conduit (passive strain $\varepsilon_{\mathrm{e}}$ ), booster pump function (active strain $\varepsilon_{\mathrm{a}}$ ), and associated strain rates (SR) in a blinded core-laboratory. The primary endpoint was the occurrence of major adverse cardiac events (MACE) 12 months post AMI. Results: RA reservoir $\left(\varepsilon_{\mathrm{s}} p=0.061\right.$, SRs $\left.p=0.049\right)$ and conduit functions $\left(\varepsilon_{\mathrm{e}} p=0.006, \mathrm{SRe}\right.$ $p=0.030)$ were impaired in patients with MACE as opposed to RA booster pump $\left(\varepsilon_{\mathrm{a}} p=0.579, \mathrm{SRa}\right.$ $p=0.118)$ and RA volume index $(p=0.866)$. RA conduit function was associated with the clinical onset of heart failure and MACE independently of RV systolic function and atrial fibrillation (AF) (multivariable analysis hazard ratio $0.95,95 \%$ confidence interval 0.92 to $0.99, p=0.009$ ), while RV systolic function and AF were not independent prognosticators. Furthermore, RA conduit strain identified low- and high-risk groups within patients with reduced RV systolic function $(p=0.019$ on
\end{abstract}


$\log$ rank testing). Conclusions: RA impairment is a distinct feature and independent risk factor in patients following AMI and can be easily assessed using CMR-FT-derived quantification of RA strain.

Keywords: myocardial infarction; risk stratification; prognosis; cardiac magnetic resonance; strain; strain rate; feature tracking; right atrium

\section{Introduction}

Cardiovascular disease, and especially myocardial infarction, are of significant clinical importance [1,2]. Left ventricular ejection fraction (LVEF) is most commonly used for risk-stratification, but does not provide information on regional cardiac function [3,4]. Recently, incremental value for LV myocardial deformation assessments has been demonstrated $[5,6]$. Myocardial strain allows precise quantification of global and regional cardiac function, and is consequently used as an endpoint in many pharmacological studies $[7,8]$. Beyond LV functional analyses, associations of left atrial involvement and cardiovascular diseases [9], as well as mortality [10], have been established following acute myocardial infarction (AMI) [11] and in heart failure with both preserved and reduced LVEF [12].

Cardiovascular magnetic resonance (CMR) represents the reference standard for cardiac morphology and function assessment $[13,14]$, including the right atrium [15]. CMR feature tracking (CMR-FT) allows the assessment of myocardial deformation based on routinely acquired balanced steady-state free precession (bSSFP) cine images. The feasibility of atrial strain assessment has been shown for both the left (LA) [16] and right (RA) [17] atrium, and may be incremental to simplistic volumetric approaches [18,19].

In contrast to evidence indicating a great role of $\operatorname{LV}[5,20]$ and LA [11,21] physiology following AMI, evidence for the impact of RA function is scarce. RA strain is associated with right atrial pressure [22,23] and with functional capacity in pulmonary arterial hypertension [22], potentially serving as a compensatory feature of impaired RV stroke volume [24]. Such compensatory impact on LV- and global cardiac function following AMI is likely but has never been adequately studied in a prospective multicenter setting. Preliminary evidence suggests a potential role for RA function in chronic heart failure [25] and in patients with coronary artery disease [15]. Consequently, the aim of the present study was the characterization of RA involvement in the clinical course following AMI.

\section{Methods}

\subsection{Study Population}

This CMR substudy includes the patients from two previously published infarction cohorts who underwent post interventional additional CMR scanning during their hospitalization. Firstly, the STEMI cohort taken from the AIDA STEMI (Abciximab Intracoronary versus intravenously Drug Application in STEMI) trial registered on ClinicalTrials.gov under NCT00712101 [26], and secondly, the NSTEMI cohort taken from the TATORT-NSTEMI (Thrombus Aspiration in Thrombus Containing Culprit Lesions in Non-ST-Elevation) trial registered with ClinicalTrials.gov under NCT01612312 [27].

The open label AIDA STEMI study enrolled 2065 patients in 22 study sites across Germany who were then randomized, comparing intracoronary $(n=1.032)$ to intravenous $(n=1.033)$ abciximab bolus application $(0.25 \mathrm{mg} / \mathrm{kg}$ bodyweight) during primary percutaneous coronary intervention (PCI). Of these, 795 patients underwent additional CMR scanning at eight of these sites, which were chosen due to their expertise.

The TATORT NSTEMI trial was conducted prospectively, enrolling 440 patients at seven study sites across Germany. Patients were randomized for either aspiration thrombectomy $(n=221)$ or standard PCI $(n=219)$, investigating the effects on microvascular injury assessed by CMR. Neither AIDA STEMI nor TATORT NSTEMI showed differences in their treatment arms. The studies were approved by both 
the lead ethical committee located at the University of Leipzig as well as all local ethical committees of involved centers. All patients gave written informed consent before randomization. The studies were conducted according to the principles of the Helsinki Declaration. The predefined CMR substudy was supported by a grant of the German Centre of Cardiovascular Research (DZHK).

\subsection{Cardiovascular Magnetic Resonance Imaging Protocol and Analyses}

All individual study sites employed one identical CMR imaging protocol on clinical 1.5 or 3.0 Tesla MR scanners [28]. CMR was conducted within the first ten days after the index event acquiring long axis 2- and 4-chamber views (CV), as well as the short axis (SA) stack. Exclusion criteria met the typical contraindications for CMR [3,28]. Myocardial infarction was characterized using LVEF, microvascular obstruction (MO), myocardial salvage, and infarct size (IS) [3,28]. RA was assessed by RA volume index (RAVI, maximal RA volume $/ \mathrm{m}^{2}$ ), as well as CMR-FT based strain analyses [17]. The dedicated feature tracking software offers a reproducible [17,29-31], fast, and detailed assessment of atrial strain $[16,17]$ in routinely-acquired b-SSFP cine images.

Briefly, strain analyses were performed by fully blinded operators on b-SSFP images using dedicated validated and established offline postprocessing software (2D CPA MR, Cardiac Performance Analysis, Version 1.1.2, TomTec Imaging Systems, Unterschleissheim, Germany) in an experienced core laboratory with excellent intra- and inter- observer reproducibility at the University Medical Center Goettingen [16,29,30,32]. First, end-diastolic RA endocardial borders were manually traced. The tracking algorithm was applied following 48 features over the cardiac cycle. Tracking accuracy was visually reviewed; where needed, corrections were made to the initial contour only. The results are based on the average of three independently repeated measurements in the 4 CV [31]. Atrial strain analyses assess the three physiological functions of atrial mechanics, i.e., the first RA reservoir function (total strain $\varepsilon_{\mathrm{s}}$ ) representing the collection of venous return during ventricular systole, the conduit function (passive strain $\varepsilon_{\mathrm{e}}$ ) for the passive early diastolic blood passage during ventricular filling, and finally, the booster pump function (active strain $\varepsilon_{\mathrm{a}}$ ) for the active late diastolic augmentation of ventricular filling (Figure 1) [16].
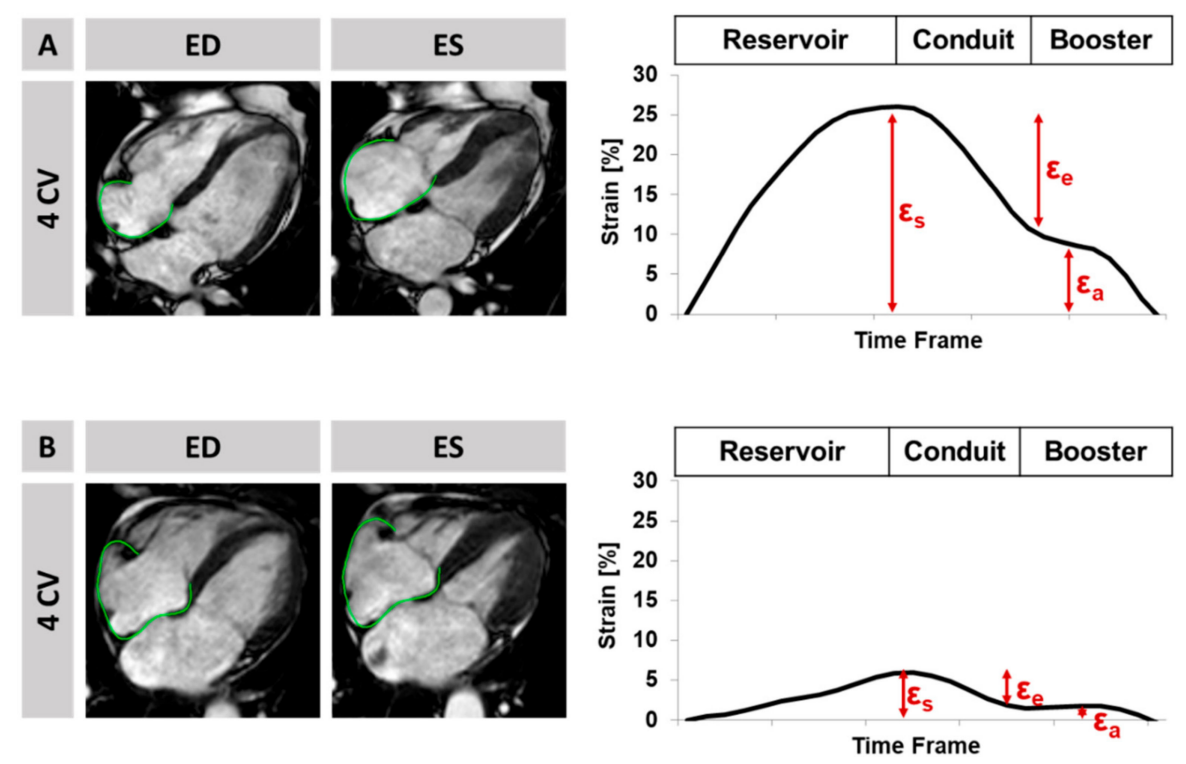

Figure 1. Feature-Tracking and Strain analysis on the left, display of a four chamber view (CV) with endocardially-tracked borders in the right atrium shown at end-diastole and systole, on the right, display of the corresponding curves of reservoir $\left(\varepsilon_{\mathrm{s}}\right)$, conduit $\left(\varepsilon_{\mathrm{e}}\right)$, and booster pump $\left(\varepsilon_{\mathrm{a}}\right)$ for a patient with (A) normal left ventricular ejection fraction of $56 \%$ and no major adverse cardiac event during follow-up, and (B) normal left ventricular ejection fraction of $52 \%$ and a major adverse clinical event during follow-up. 


\subsection{Clinical Endpoints}

The primary clinical endpoint of this study was the occurrence of major adverse cardiovascular events (MACE) including all-cause death, reinfarction, or congestive heart failure during the first year after the initial event. To avoid statistical falsification, each patient could only account for one event according to the respective severity (death $>$ reinfarction $>$ congestive heart failure). A blinded clinical endpoints committee adjudicated all components of the combined clinical endpoints on the basis of data provided by the clinical trial sites. Detailed patient outcome and definitions have been published previously $[26,27,33]$.

\subsection{Statistics}

Statistical calculations were performed using IBM SPSS Statistic Software Version 24 for Windows (IBM, Armonk, NY, USA), R version 3.3.2 (R Core Development Team, Vienna, Austria) and RStudio version 1.0.44 (RStudio Inc., Boston, MA, USA). All $p$-values provided are two-sided; an alpha level of 0.05 and below was considered statistically significant. Categorical parameters are reported in absolute numbers and related percentage values. Continuous parameters are presented as median with interquartile range (IQR). Differences between categorical variables were compared using the chi-square test. Independent continuous parameters were compared using the Mann-Whitney-U test. Continuous parameters were checked for normal distribution based on the Shapiro-Wilk test. The correlation of non-normally distributed parameters was evaluated using the Spearman's rank correlation coefficient. Clinical endpoint analyses were performed using Kaplan-Meier plots and associated log-rank tests. Linear univariate Cox regression was used for calculation of hazard ratios (HR) which are given with corresponding $95 \%$ confidence intervals (CI). Multivariable Cox regression models were then performed for further statistical adjustments of univariate significant factors. Reproducibility was tested within 30 (15 STEMI, 15 NSTEMI) randomly selected patients. To assess intraobserver variability, these cases were analyzed twice, with at least four weeks in between repeated analyses by a blinded operator. For interobserver variability, these cases were analyzed by a second blinded operator. Calculations comprised Bland-Altman analysis [34], intraclass correlation coefficients (ICC), and coefficients of variation $(\mathrm{CoV}$, standard deviation of the differences divided by the mean). Agreements are considered excellent ICC $>0.74$, good 0.60-0.74, fair 0.4-0.59, or poor $<0.4$ [30].

\section{Results}

\subsection{Study Population}

The study population, with a median age of 64 years $(53,72)$, consisted of 1235 patients, of which $n=795$ were recruited in AIDA STEMI and $n=440$ in TATORT NSTEMI. CMR, performed in median on day three (IQR 2-4) after the index event, was available in 1031 patients with regards to complete imaging protocols and image quality for FT postprocessing (Figure 2). Follow-up was achieved in $99.8 \%$ of all patients with available FT data. Of these patients, 71 had a predefined event within the first year after infarction (death $n=29$, reinfarction $n=25$ and/or readmission due to congestive heart failure $n=35$ ).

Baseline characteristics and comparisons between MACE and no MACE are reported in Table 1. Patients with predefined MACE were significantly older $(p<0.001)$, suffered more frequently from hypertension $(p=0.019)$, and smoking was less common amongst them $(p=0.039)$. Killip class on admission $(p<0.001)$ and the number of diseased vessels $(p=0.025)$ were significantly worse in patients with MACE, whilst time to treatment, the affected artery, pre- and post- interventional TIMI flow, as well as number of implanted stents were similar. 


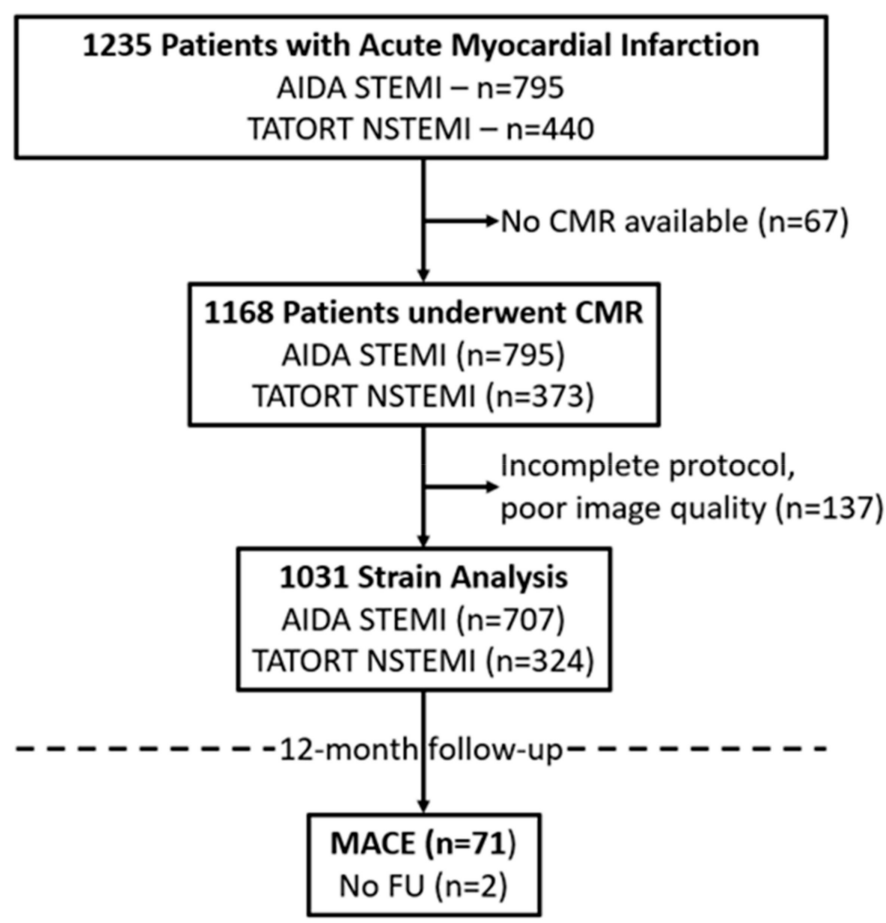

Figure 2. Study Flow-Chart. CMR = cardiovascular magnetic resonance, $\mathrm{MACE}=$ major adverse cardiovascular events, N/STEMI = non/ ST-elevation myocardial infarction.

Table 1. Baseline characteristics.

\begin{tabular}{|c|c|c|c|c|}
\hline Variable & All Patients $n=1031$ & MACE $n=71$ & No MACE $n=960$ & $p$-Value \\
\hline Age & $64(53,72)$ & $71(60,77)$ & $63(52,72)$ & $<0.001$ \\
\hline $\operatorname{Sex}(m)$ & $774 / 1029(75.2 \%)$ & $47 / 71(66.2 \%)$ & $727 / 958(75.9 \%)$ & 0.068 \\
\hline \multicolumn{5}{|l|}{$\begin{array}{l}\text { Cardiovascular } \\
\text { risk factors }\end{array}$} \\
\hline Active smoking & 415/951 (43.6\%) & $20 / 64(31.3 \%)$ & $395 / 887(44.6 \%)$ & 0.039 \\
\hline Hypertension & $728 / 1027(70.6 \%)$ & $59 / 71(83.1 \%)$ & $669 / 956(70 \%)$ & 0.019 \\
\hline Hyperlipoproteinemia & $387 / 1024(37.8 \%)$ & $25 / 71(35.2 \%)$ & $362 / 953(38 \%)$ & 0.642 \\
\hline Diabetes & $243 / 1027(23.7 \%)$ & $23 / 71(32.4)$ & $220 / 956(23 \%)$ & 0.073 \\
\hline $\begin{array}{l}\text { Body mass index } \\
\qquad\left(\mathrm{kg} / \mathrm{m}^{2}\right)\end{array}$ & $27.5(24.9,30.4)$ & $27.7(25.5,31.1)$ & $27.5(24.9,30.3)$ & 0.433 \\
\hline Previous & & & & \\
\hline $\begin{array}{l}\text { myocardial } \\
\text { infarction }\end{array}$ & $72 / 1027(7 \%)$ & $4 / 71(5.6 \%)$ & $68 / 956(7.1 \%)$ & 0.638 \\
\hline Previous PCI & $89 / 1028(8.7 \%)$ & $5 / 71(7 \%)$ & $84 / 957(8.8 \%)$ & 0.616 \\
\hline Previous CABG & $19 / 1028(1.8 \%)$ & $2 / 69(2.9 \%)$ & $17 / 957(1.8 \%)$ & 0.530 \\
\hline $\begin{array}{c}\text { ST-segment } \\
\text { elevation }\end{array}$ & $707 / 1029(68.7 \%)$ & $48 / 71(67.6 \%)$ & $659 / 958(68.8 \%)$ & 0.836 \\
\hline $\begin{array}{c}\text { Systolic blood } \\
\text { pressure }(\mathrm{mmHg})\end{array}$ & $133(119,150)$ & $130(110,150)$ & $134(120,150)$ & 0.166 \\
\hline $\begin{array}{l}\text { Diastolic blood } \\
\text { pressure }(\mathrm{mmHg})\end{array}$ & $80(70,89)$ & $77(65,85)$ & $80(70,89)$ & 0.059 \\
\hline $\begin{array}{l}\text { Heart rate } \\
\text { (beats/min) }\end{array}$ & $76(67,86)$ & $80(70,96)$ & $76(66,86)$ & 0.001 \\
\hline $\begin{array}{l}\text { Time symptoms to } \\
\text { balloon * }(\mathrm{min})\end{array}$ & $180(110,310)$ & $194(114,390)$ & $180(109,306)$ & 0.279 \\
\hline $\begin{array}{c}\text { Door-to-balloon } \\
\text { time* }(\mathrm{min})\end{array}$ & $30(22,42)$ & $28(22.5,40)$ & $30(22,42)$ & 0.490 \\
\hline
\end{tabular}


Table 1. Cont.

\begin{tabular}{|c|c|c|c|c|}
\hline Variable & All Patients $n=1031$ & MACE $n=71$ & No MACE $n=960$ & $p$-Value \\
\hline $\begin{array}{l}\text { Killip class on } \\
\text { admission }\end{array}$ & & & & $<0.001$ \\
\hline 1 & $911 / 1029$ (88.7\%) & $46 / 71(64.8 \%)$ & $865 / 958(90.3 \%)$ & \\
\hline 2 & $81 / 1029(7.9 \%)$ & $16 / 71(22.5 \%)$ & $65 / 958(6.8 \%)$ & \\
\hline 3 & $22 / 1029(2.1 \%)$ & $5 / 71(7 \%)$ & $17 / 958(1.8 \%)$ & \\
\hline 4 & $15 / 1029(1.5 \%)$ & $4 / 71(5.6 \%)$ & $11 / 958(1.1 \%)$ & \\
\hline Diseased vessels & & & & 0.025 \\
\hline 1 & $517 / 1029(50.3 \%)$ & $27 / 71(38 \%)$ & $490 / 958(51.1 \%)$ & \\
\hline 2 & $311 / 1029(30.3 \%)$ & $22 / 71(31 \%)$ & $289 / 958(30.2 \%)$ & \\
\hline 3 & $201 / 1029(19.6 \%)$ & $22 / 71(31 \%)$ & $179 / 958(18.7 \%)$ & \\
\hline Affected artery & & & & 0.337 \\
\hline $\begin{array}{l}\text { left anterior } \\
\text { descending }\end{array}$ & $425 / 1029(41.4 \%)$ & $37 / 71(52.1 \%)$ & $388 / 958$ (40.5\%) & \\
\hline left circumflex & $213 / 1029(20.7 \%)$ & $14 / 71(19.7 \%)$ & $199 / 958(20.8 \%)$ & \\
\hline left main & $4 / 1029(0.4 \%)$ & $0 / 71(0 \%)$ & $4 / 958(0.4 \%)$ & \\
\hline $\begin{array}{l}\text { right coronary } \\
\text { artery }\end{array}$ & $381 / 1029(37.1 \%)$ & $20 / 71(28.2 \%)$ & $361 / 958(37.7 \%)$ & \\
\hline bypass graft & $6 / 1029(0.6 \%)$ & $0 / 71(0 \%)$ & 6/958 (0.6\%) & \\
\hline $\begin{array}{l}\text { TIMI flow grade } \\
\text { before PCI }\end{array}$ & & & & 0.607 \\
\hline 0 & $515 / 1029(50.1 \%)$ & $40 / 71(56.3 \%)$ & $475 / 958(49.6 \%)$ & \\
\hline 1 & $111 / 1029(10.8 \%)$ & $5 / 71(7 \%)$ & $106 / 958(11.1 \%)$ & \\
\hline 2 & $215 / 1029(20.9 \%)$ & $13 / 71(18.3 \%)$ & $202 / 958(21.1 \%)$ & \\
\hline 3 & $188 / 1029(18.3 \%)$ & $13 / 71(18.3 \%)$ & $175 / 958(18.3 \%)$ & \\
\hline Stent implanted & $1005 / 1029(97.9 \%)$ & $69 / 71(97.2 \%)$ & $936 / 958(97.7 \%)$ & 0.524 \\
\hline $\begin{array}{l}\text { TIMI flow grade } \\
\text { after PCI }\end{array}$ & & & & 0.294 \\
\hline 0 & $20 / 1029(1.9 \%)$ & $1 / 71(1.4 \%)$ & $19 / 958(2 \%)$ & \\
\hline 1 & $21 / 1029(2.0 \%)$ & $3 / 71(4.2 \%)$ & $18 / 958(1.9 \%)$ & \\
\hline 2 & $76 / 1029(7.4 \%)$ & $8 / 71(11.3 \%)$ & $68 / 958(7.1 \%)$ & \\
\hline 3 & $912 / 1029(88.8 \%)$ & $59 / 71(83.1 \%)$ & $853 / 958(89 \%)$ & \\
\hline \multicolumn{5}{|l|}{ Medication } \\
\hline $\begin{array}{l}\text { Glycoprotein } \\
\mathrm{IIb} / \mathrm{III} \text { inhibitor }\end{array}$ & 729/1028 (70.9\%) & $51 / 71(71.8 \%)$ & $678 / 957(70.8 \%)$ & 0.860 \\
\hline Aspirin & 1027/1029 (99.8\%) & $71 / 71(100 \%)$ & 956/958 (99.8\%) & 0.700 \\
\hline $\begin{array}{l}\text { Clopidogrel/Prasugrel/ } \\
\text { Ticagrelor }\end{array}$ & $1028 / 1028(100 \%)$ & $71 / 71(100 \%)$ & 957/957 (100\%) & \\
\hline Betablocker & $983 / 1028(95.6 \%)$ & $69 / 71(97.2 \%)$ & 914/957 (95.5\%) & 0.505 \\
\hline $\begin{array}{l}\text { ACE-inhibitor/AT-1 } \\
\text { antagonist }\end{array}$ & $947 / 1028(92.1 \%)$ & $67 / 71(94.4 \%)$ & $880 / 957$ (92\%) & 0.467 \\
\hline $\begin{array}{c}\text { Aldosterone } \\
\text { antagonist }\end{array}$ & $133 / 1028(12.9 \%)$ & 23/71 (32.4\%) & 110/957 (11.5\%) & $<0.001$ \\
\hline Statin & $990 / 1028(96.3 \%)$ & $69 / 71(97.2 \%)$ & $921 / 957(96.2 \%)$ & 0.684 \\
\hline Time to MRI (days) & $3(2,4)$ & $3(2,4)$ & $3(2,4)$ & 0.06 \\
\hline
\end{tabular}

Data presented as $\mathrm{n} / \mathrm{N}(\%)$ or median (IQR). $p$-values were calculated for the comparison between patients with and without MACE. Numbers in bold type indicate a significant difference. ${ }^{*}$ only assessed in STEMI patients $(n=$ 795), $\mathrm{CABG}=$ coronary artery bypass graft; $\mathrm{MACE}=$ major adverse cardiac event; $\mathrm{PCI}=$ percutaneous coronary intervention; TIMI $=$ Thrombolysis In Myocardial Infarction. $p$-values in bold indicate statistical significance $<0.05$.

Overall, patients had a preserved LVEF of 50.5\% (IQR 43.5\%, 57.5\%) and RVEF of 61.1\% (IQR $54.2 \%, 67.7 \%$ ), an IS of $13.1 \%$ LV (IQR 5.2\%, 21.7\%) with an area at risk of $29.1 \% \mathrm{LV}$ (IQR 20.1\%, 42.2\%), a myocardial salvage index of $54.7 \%$ (IQR $34.8 \%, 74.9 \%$ ), and a microvascular obstruction of $0.33 \% \mathrm{LV}$ 
(IQR 0\%, 1.9\%) as assessed by CMR. Of these, LVEF $(p<0.001)$, RVEF $(p=0.037)$, IS $(p=0.001)$ and myocardial salvage index $(p=0.032)$ were significantly more impaired in patients with MACE, while MO $(p=0.06)$ and area at risk $(p=0.08)$ did not reach statistical significance.

\subsection{Right Atrial Dysfunction}

CMR-derived RA functional parameters are reported in Table 2, and RA strain reproducibility is shown in Table 3. RA conduit function showed the most significant difference between patients with and without MACE $\left(\varepsilon_{\mathrm{e}} p=0.006\right.$, SRe $\left.p=0.03\right)$, followed by reservoir function $\left(\varepsilon_{\mathrm{s}} p=0.061\right.$, SRs $p=0.049)$. Booster pump function was similar in both groups $\left(\varepsilon_{\mathrm{a}} p=0.579\right.$, SRa $\left.p=0.118\right)$ (Table 2). Similarly, discrimination between patients admitted for rehospitalization due to heart failure was best for conduit $\left(\varepsilon_{\mathrm{e}} p=0.003\right.$, SRe $\left.p=0.008\right)$, followed by reservoir $\left(\varepsilon_{\mathrm{s}} p=0.029\right.$, SRs $\left.p=0.087\right)$ function. Booster pump strain did not provide risk discrimination $\left(\varepsilon_{\mathrm{a}} p=0.909\right.$, SRa $\left.p=0.367\right)$. Conduit $\left(\varepsilon_{\mathrm{e}} p<0.001\right.$, SRe $\left.p=0.007\right)$ but not reservoir $\left(\varepsilon_{\mathrm{S}} p=0.068\right.$, SRs $\left.p=0.709\right)$ or booster pump function $\left(\varepsilon_{\mathrm{a}} p=0.341\right.$, SRa $\left.p=0.148\right)$ discriminated between patients without (Killip class $\left.=1\right)$ and with clinical signs of heart failure (Killip class $\geq 2$ ). Body surface area (BSA)-indexed maximum RA volumes were not significantly different between patients with and without MACE $(p=0.866)$ (Table 2$)$ or heart failure $(p=0.758)$. RA function was significantly decreased in patients with atrial fibrillation (AF) $\left(\varepsilon_{\mathrm{s}} p<0.001, \varepsilon_{\mathrm{e}} p<0.001\right.$ and $\left.\varepsilon_{\mathrm{a}} p=0.022\right)$.

RA function as assessed by strain and SR parameters did not correlate with IS ( $p>0.167$ for all). Correlation between RA performance and RVEF was weak but highly significant for all atrial functional parameters (RA $\varepsilon_{\mathrm{S}} r=0.19$, SRs $r=0.23, \varepsilon_{\mathrm{e}} r=0.15$, SRe $r=-0.17, \varepsilon_{\mathrm{a}} r=0.14$, and SRa -0.13 , $p<0.001$ for all). Similarly, correlation with LVEF was weak and strongly significant for reservoir and conduit function (RA $\varepsilon_{\mathrm{S}} r=0.14$, SRs $r=0.13, \varepsilon_{\mathrm{e}} r=0.19$ and SRe $r=-0.15, p<0.001$ for all), while booster pump function did not correlate with $\operatorname{LVEF}\left(\varepsilon_{\mathrm{a}} r=0.06, p=0.073\right.$ and SRa $\left.-0.05, p=0.104\right)$.

Table 2. Atrial Performance.

\begin{tabular}{|c|c|c|c|c|}
\hline & All Patients & MACE & No MACE & \\
\hline Functional parameter & median (IQR) & median (IQR) & median (IQR) & $p$ \\
\hline \multicolumn{5}{|l|}{ Infarct characteristics } \\
\hline IS & $13.1(5.20,21.7)$ & $20.3(9.83,28.9)$ & $12.8(5.15,21.3)$ & 0.001 \\
\hline AAR & $29.1(20.1,42.2)$ & $32.9(24.2,45.1)$ & $28.8(20.0,42.0)$ & 0.080 \\
\hline $\mathrm{MO}$ & $0.33(0.00,1.92)$ & $0.80(0.00,2.53)$ & $0.29(0.00,1.90)$ & 0.060 \\
\hline \multicolumn{5}{|l|}{ Left ventricle } \\
\hline LV mass & $66.1(57.4,75.9)$ & $68.9(58.9,78.7)$ & $65.9(57.2,75.8)$ & 0.380 \\
\hline EDV & $73.3(62.5,86.0)$ & $75.4(67.0,87.5)$ & $73.1(62.1,85.8)$ & 0.155 \\
\hline ESV & $35.6(27.8,45.9)$ & $45.1(31.6,54.1)$ & $35.2(27.6,45.3)$ & $<0.001$ \\
\hline EF & $50.5(43.5,57.5)$ & $41.2(33.0,52.2)$ & $51.0(44.5,57.6)$ & $<0.001$ \\
\hline Strain & $-16.6(-12.5,-20.2)$ & $-11.7(-8.18,-17.1)$ & $-16.8(-13.0,-20.4)$ & $<0.001$ \\
\hline \multicolumn{5}{|l|}{ Left atrium } \\
\hline LAVI & $35.0(26.6,44.3)$ & $40.6(28.7,53.6)$ & $34.6(26.6,43.4)$ & 0.001 \\
\hline LA Es & $20.9(16.2,25.7)$ & $16.2(11.4,21.1)$ & $21.2(16.7,26.1)$ & $<0.001$ \\
\hline LA Ee & $8.69(5.63,11.7)$ & $6.92(3.19,8.73)$ & $8.83(5.83,11.9)$ & $<0.001$ \\
\hline LA Ea & $11.5(8.60,15.3)$ & $9.96(5.91,12.7)$ & $11.7(8.77,15.5)$ & $<0.001$ \\
\hline LA SRs & $0.88(0.70,1.08)$ & $0.79(0.59,0.93)$ & $0.90(0.71,1.10)$ & $<0.001$ \\
\hline LA SRe & $-0.55(-0.38,-0.78)$ & $-0.48(-0.34,-0.67)$ & $-0.56(-0.39,-0.79)$ & 0.004 \\
\hline LA SRa & $-0,96(-0.73,-1.25)$ & $-0.84(-0.59,-1.06)$ & $-0.97(-0.73,-1.26)$ & 0.001 \\
\hline \multicolumn{5}{|l|}{ RV volumes } \\
\hline RV mass & $22.2(18.9,26.2)$ & $20.8(19.4,24.9)$ & $22.2(18.9,26.4)$ & 0.247 \\
\hline EDV & $60.9(51.3,71.4)$ & $59.8(48.0,68.2)$ & $61.0(51.6,71.5)$ & 0.122 \\
\hline ESV & $23.1(17.4,31.2)$ & $23.1(16.3,35.6)$ & $23.1(17.5,30.8)$ & 0.878 \\
\hline $\mathrm{EF}$ & $61.1(54.2,67.7)$ & $56.5(46.1,69.4)$ & $61.3(54.6,67.7)$ & 0.037 \\
\hline
\end{tabular}


Table 2. Cont.

\begin{tabular}{ccccc}
\hline & All Patients & MACE & No MACE & \\
\hline Right atrium & & & & \\
RAVI & $27.4(20.7,35.7)$ & $26.7(18.7,36.3)$ & $27.4(20.8,35.6)$ & 0.866 \\
RA Es & $24.4(17.3,32.4)$ & $22.1(13.9,30.7)$ & $24.8(17.5,32.5)$ & 0.061 \\
RA Ee & $10.9(6.03,16.5)$ & $8.88(3.99,13.9)$ & $11.1(6.18,16.7)$ & $\mathbf{0 . 0 0 6}$ \\
RA Ea & $12.3(7.89,17.5)$ & $11.4(6.31,18.6)$ & $12.3(8,17.4)$ & 0.579 \\
RA SRs & $1.11(0.83,1.43)$ & $0.98(0.64,1.43)$ & $1.11(0.84,1.43)$ & $\mathbf{0 . 0 4 9}$ \\
RA SRe & $-0.54(-0.33,-0.79)$ & $-0.48(-0.21,-0.65)$ & $-0.55(-0.34,-0.8)$ & $\mathbf{0 . 0 3 0}$ \\
RA SRa & $-0.96(-0.66,-1.37)$ & $-0.89(-0.52,-1.28)$ & $-0.97(-0.67,-1.37)$ & 0.118 \\
\hline
\end{tabular}

$\mathrm{IQR}=$ interquartile range, $\mathrm{IS}=$ infarct size, $\mathrm{AAR}=$ area at risk, $\mathrm{MO}=$ microvascular obstruction, $\mathrm{RA} / \mathrm{V}=$ right atrium/ventricle, Es/SRs = reservoir strain/rate, Ee/SRe = conduit strain/rate and Ea/SRa = booster pump strain/rate, $\mathrm{LAVI} / \mathrm{RAVI}=$ left/right atrial volume index. $p$-values in bold indicate statistical significance $<0.05$.

Table 3. Reproducibility of right atrial strain assessment.

\begin{tabular}{cccc}
\hline & Mean Difference \pm SD (\%) & CoV (\%) & ICC (95\% CI) \\
\hline Intraobserver & & & \\
\hline RA Es & $-2.05 \pm 3.84$ & 13.73 & $0.95(0.86-0.98)$ \\
RA Ee & $-0.17 \pm 2.23$ & 16.95 & $0.97(0.94-0.99)$ \\
RA Ea & $-1.98 \pm 4.58$ & 30.84 & $0.83(0.62-0.92)$ \\
RA SRs & $-0.08 \pm 0.32$ & 26.02 & $0.82(0.63-0.92)$ \\
RA SRe & $0.02 \pm 0.17$ & 31.48 & $0.91(0.80-0.96)$ \\
RA SRa & $0.08 \pm 0.29$ & 25.66 & $0.85(0.69-0.93)$ \\
\hline Interobserver & & & \\
RA Es & $1.67 \pm 6.23$ & 23.86 & $0.87(0.72-0.94)$ \\
RA Ee & $1.22 \pm 4.14$ & 33.15 & $0.88(0.74-0.94)$ \\
RA Ea & $0.44 \pm 3.44$ & 25.26 & $0.93(0.84-0.97)$ \\
RA SRs & $0.01 \pm 0.29$ & 25.44 & $0.84(0.66-0.93)$ \\
RA SRe & $0.02 \pm 0.20$ & 37.04 & $0.88(0.74-0.94)$ \\
RA SRa & $-0.03 \pm 0.29$ & 27.10 & $0.86(0.70-0.93)$ \\
\hline
\end{tabular}

$\mathrm{SD}=$ standard deviation, $\mathrm{CoV}=$ coefficient of variation, ICC = intraclass correlation coefficient, RA Es/e/a and $\mathrm{SRs} / \mathrm{e} / \mathrm{a}=$ right atrial reservoir, conduit and booster pump strain, and associated strain rate.

\subsection{Risk Stratification}

In univariate Cox regression (Table 4), only RA conduit function had a highly statistically significant impact on MACE occurrence (HR 0.95, 95\% CI 0.91 to $0.98, p=0.003$ ), while reservoir strain did not reach statistical significance (HR $0.98,95 \%$ CI 0.96 to $1.00, p=0.062$ ). Booster pump function did not predict MACE (HR 1.00, 95\% CI 0.98 to $1.04, p=0.745$ ). Similarly, RAVI did not impact outcome (HR 1.01, 95\% CI 0.99-1.02, $p=0.634$ ). AF was significantly associated with the incidence of MACE (HR 2.25, 95\% CI 1.20-4.23, $p=0.011$ ) (Table 3). Considering heart failure separately regarding outcome both conduit $\left(\varepsilon_{\mathrm{e}} p=0.003\right.$, SRe $\left.p=0.008\right)$ and reservoir functions $\left(\varepsilon_{\mathrm{s}} p=0.029\right)$ were impaired in these patients and identified increased risk ( $\varepsilon_{\mathrm{e}} \mathrm{HR} 0.92,95 \%$ CI 0.87 to $0.97, p=0.004, \varepsilon_{\mathrm{s}}$ HR $0.96,95 \%$ CI 0.93 to $1.00, p=0.027$ ). Booster pump had no impact (HR $1.0095 \%$ CI 0.95 to $1.04, p=0.830$ ).

In multivariable Cox-regression analyses including RVEF, AF, and passive conduit function (Ee) using continuous parameters, passive conduit function revealed independence, irrespective of RVEF and AF for MACE (RA $\varepsilon_{\mathrm{e}}$ HR $0.95,95 \%$ CI 0.92 to $0.99, p=0.009$ ), whilst RVEF (RVEF HR 0.98. 95\% CI 0.96-1.00, $p=0.085$ ) and AF (HR 1.59, 95\% CI 0.75-3.40, $p=0.227$ ) did not emerge as independent prognostic factors. A trend remained for passive conduit RA function (Ee, HR 0.97, 95\% CI 0.93-1.01, $p=0.088$ ) if LV EF was additionally to be included (LVEF, HR 0.94, 95\% CI 0.92-0.97, $p<0.001$ ).

Kaplan-Meier Plots and associated Log-rank testing revealed a significant impact of atrial function on MACE occurrence $\left(\varepsilon_{\mathrm{s}}\right.$ cut off $23.17 \%, p=0.024 ; \varepsilon_{\mathrm{e}}$ cut off $16.13 \%, p=0.003$ and $\varepsilon_{\mathrm{a}}$ cut off $6.43 \%$, 
$p=0.008$ ) after ROC optimized dichotomization (Figure 3). Additionally, atrial function discriminated elevated risks for patients with RVEF below the median (Figure 4).

Table 4. Predictors of MACE in univariable Cox regression analysis.

\begin{tabular}{ccc}
\hline Variable & Univariable Hazard Ratio (CI) & $p$ \\
\hline Age & $1.04(1.02-1.06)$ & $<\mathbf{0 . 0 0 1}$ \\
Hypertension & $2.07(1.11-3.84)$ & $\mathbf{0 . 0 2 2}$ \\
LVEF & $0.94(0.92-0.96)$ & $<\mathbf{0 . 0 0 1}$ \\
RVEF & $0.97(0.95-0.99)$ & $\mathbf{0 . 0 1 2}$ \\
Infarct Size & $1.03(1.01-1.05)$ & $\mathbf{0 . 0 0 1}$ \\
Killip Class & $2.08(1.64-2.64)$ & $<\mathbf{0 . 0 0 1}$ \\
Number of diseased vessels & $1.46(1.10-1.94)$ & $\mathbf{0 . 0 0 9}$ \\
Atrial fibrillation & $2.25(1.20-4.23)$ & $\mathbf{0 . 0 1 1}$ \\
RA-Es & $0.98(0.96-1.00)$ & 0.062 \\
RA-Ee & $0.95(0.91-0.98)$ & $\mathbf{0 . 0 0 3}$ \\
RA-Ea & $1.00(0.98-1.04)$ & 0.745 \\
RAVI & $1.01(0.99-1.02)$ & 0.634
\end{tabular}

$\mathrm{CI}=$ confidence interval, LV/RF-EF $=$ left/right ventricular ejection fraction, $\mathrm{RA}=$ right atrium, Es $=$ reservoir strain, $\mathrm{Ee}=$ conduit strain, $\mathrm{Ea}=$ booster pump strain, and RAVI = right atrial volume index. $p$-values in bold indicate statistical significance $<0.05$.
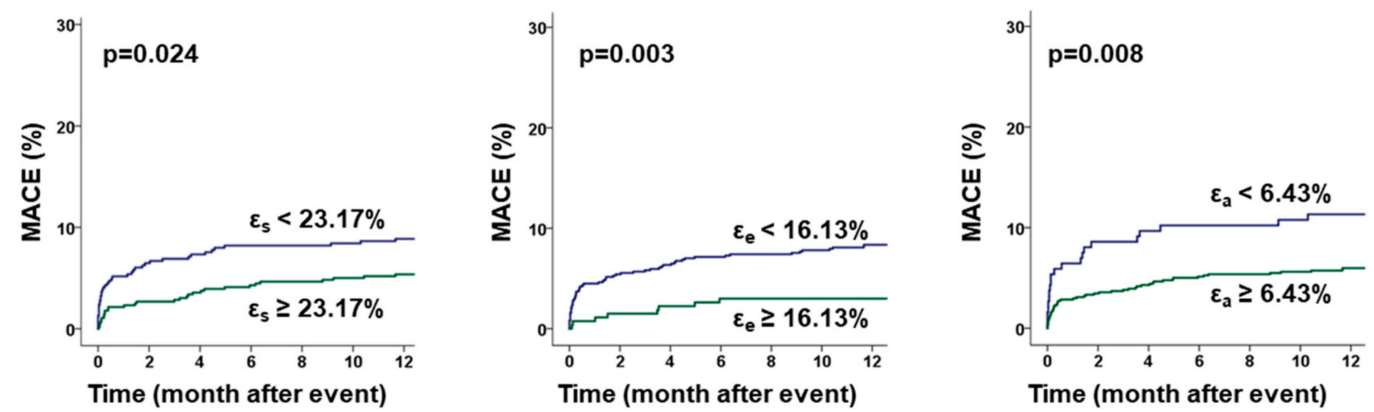

Figure 3. Influence of RA Strain on MACE prediction. The graphs show the influence of right atrial (RA) reservoir $\left(\varepsilon_{\mathrm{s}}\right)$, conduit $\left(\varepsilon_{\mathrm{e}}\right)$ and booster pump $\left(\varepsilon_{\mathrm{a}}\right)$ strain on the rate of major adverse clinical events (MACE) during 12 months follow-up after ROC-adapted dichotomization, $p$ values calculated by log-rank test.
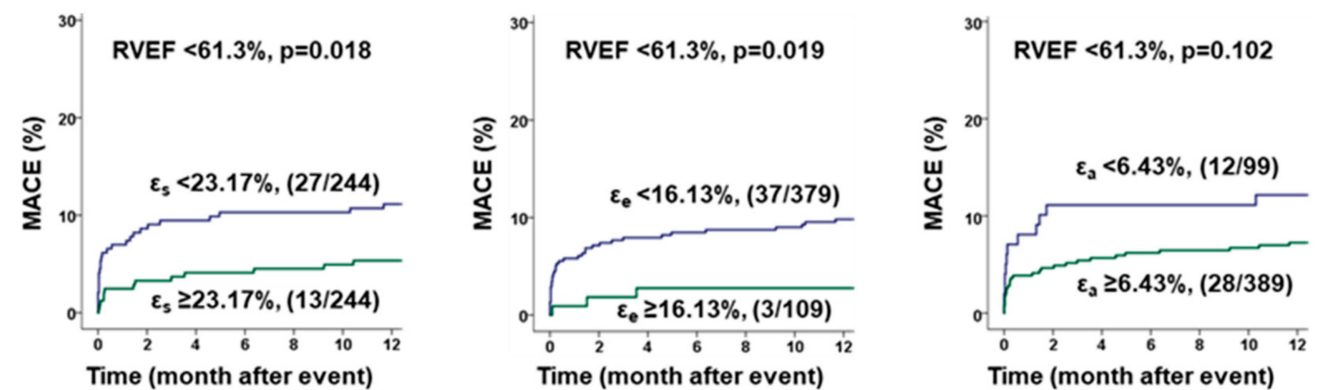

Figure 4. Benefit of additional right atrial strain analysis over sole right ventricular function. The graphs show the impact of right atrial (RA) reservoir $\left(\varepsilon_{\mathrm{s}}\right)$, conduit $\left(\varepsilon_{\mathrm{e}}\right)$ and booster pump $\left(\varepsilon_{\mathrm{a}}\right)$ function evaluation on the occurrence of major adverse cardiac events (MACE). RA function was investigated in addition to right ventricular ejection fraction (RVEF) below the median. Cut-Offs for RA strains were chosen for optimal sensitivity and specificity, and $p$ values were calculated by log-rank test for the occurrence of major adverse clinical events (MACE) within 12 months after myocardial infarction. 


\section{Discussion}

The present study reports the value of CMR-FT derived data on RA performance following AMI. The study bears several notable findings regarding RA function and associated passive and active phases. RA passive conduit function reveals the highest association with MACE. Passive atrial restoring forces are associated with outcome independently of RV systolic function. Furthermore, they are associated with clinical onset of heart failure symptoms. Although there is a significant correlation of atrial functional phases and both right and left ventricular systolic function, correlation is low, and indicates a significant independent contribution of RA performance beyond systolic biventricular function.

Impaired conduit function showed the highest association with increased risk for adverse clinical events. A decrease of RA passive emptying fraction has been shown for patients with increased pulmonary artery pressure [35], coronary slow-flow [36], and left ventricular dysfunction related to left ventricular hypertrophy [37] and heart failure with preserved ejection fraction (HFpEF) [38]. On the one hand, RA conduit function is connected to and reflects right and left ventricular (dys) function, as described by a significant correlation of passive conduit function with systolic RV and LV function. RA conduit strain is strongly related to early RV filling in HFpEF [38], thus contributing to $\mathrm{RV}$ and, subsequently, LV stroke volumes. On the other hand, correlation was low indicating value of atrial function beyond systolic biventricular function. At part, impaired RA conduit function may also imply diastolic RV dysfunction. The onset of diastolic dysfunction forestalls systolic dysfunction during the ischemic cascade [39], and a link between total LA strain and LV filling pressures has previously been made [40]. Reports also indicate independence of LA passive conduit function of LV stiffness [41]; thus, RA passive restoring forces (conduit function) themselves may have a distinct role in cardiac pathophysiology and patients with AMI beyond RV function. Indeed, the value of RA conduit function grows beyond sole RV function. In HFpEF, RA conduit function showed strong associations with maximum oxygen uptake independent of sole RV stiffness and relaxation [38]. Impaired RA function was significantly associated with heart failure symptoms as assessed using Killip class and heart failure readmission. AF was associated with reduced atrial function, including all three atrial functional phases. Indeed, impaired RA strain is a known predictor of AF reoccurrence in paroxysmal AF [42]. Importantly, passive conduit function provided risk stratification for MACE independently of systolic RV function and AF, whilst RVEF and AF were not independently associated with outcome. Additionally, RA conduit function provided incremental value for risk stratification in patients presenting with RVEF below the median. However, the impact of RA function on MACE occurrence was not independent of LV function, as shown by multivariable analyses with only a trend remaining for RA Ee.

Quantification of total RA function is challenging [43]. It is noteworthy that the overall reservoir/total strain values assessed in this study are similar to previously published reference values [44], underlining consistency. Total LA strain is an independent risk factor for MACE beyond LV function [11], and is able to compensate for LV heart failure in left anterior descending lesions [45]. RA reservoir strain was significantly associated with increased risk for heart failure and after ROC-adapted dichotomization reservoir function identified elevated risk for MACE in Kaplan-Meier plots, providing incremental value for risk stratification in patients with RVEF below the median. On the one hand side, impaired RA function leads to impaired systolic RV function and, subsequently, LV preload and cardiac output [46]. However, in contrast to passive atrial function reflecting early RV filling and elastic atrial restoring forces, RA reservoir function is associated with pulmonary artery pressures caused by LV heart failure [47], and may rather reflect the degree of congestion. Since BSA-indexed RA volumes were similar comparing patients with and without MACE, this fact could explain why passive atrial strain is distinctly more precise in the identification of patients at risk compared to total atrial strain. 


\section{Limitations}

Several limitations need to be addressed for accurate interpretation of the findings. First, this CMR substudy included patients from the multicenter AIDA STEMI and TATORT NSTEMI trials; thus, CMR was performed in eight centers using different $C M R$ vendors and sequences. However, the study sites were chosen for their expertise and employed the same imaging protocol. Furthermore, data analyses were performed in one experienced core-laboratory. Second, due to the acute nature of AMI and diverse impacts on each patient, the optimal time-point of CMR may vary. While with a median of three (IQR 2-4) days a consistent time point was achieved, little is known about the optimal time-point for CMR and its impact on risk stratification. Additionally, sicker patients potentially underwent CMR later or not at all, resulting in a possible selection bias. However, a large number of patients, i.e., 1031, post PCI has been prospectively enrolled shortly after intervention, compared to only 67 patients who did not undergo CMR imaging. Lastly, data on estimated systolic pulmonary artery pressure, which may influence RA function, was not systematically assessed, and is consequently not available.

\section{Conclusions}

RA function is associated with onset of clinical heart failure symptoms and outcome. Amongst the three atrial functional phases, RA passive conduit function most precisely identifies the degree of heart failure, as well as patients at risk for MACE. RA conduit function adds incremental prognostic value beyond the assessment of systolic ventricular function following AMI, suggesting an important contribution to global cardiac mechanics.

Author Contributions: A.S., S.J.B., H.T., and I.E. designed the study protocol, performed data acquisition, performed statistical analyses and drafted the manuscript. J.-L.N., A.K. and T.S. performed data acquisition/analysis and revised the manuscript. J.U. performed statistical analyses. T.S., K.-P.R., J.T.K., B.B., S.K., M.G., G.H. and revised the manuscript and participated in the scientific discussion during the study. All authors read and approved the final manuscript.

Funding: This research was funded by the German Center for Cardiovascular Research (DZHK).

Conflicts of Interest: The authors declare no conflict of interest.

\section{References}

1. Smith, S.C.; Collins, A.; Ferrari, R.; Holmes, D.R.; Logstrup, S.; McGhie, D.V.; Ralston, J.; Sacco, R.L.; Stam, H.; Taubert, K.; et al. Our time: A call to save preventable death from cardiovascular disease (heart disease and stroke). Circulation 2012, 126, 2769-2775. [CrossRef] [PubMed]

2. Roffi, M.; Patrono, C.; Collet, J.-P.; Mueller, C.; Valgimigli, M.; Andreotti, F.; Bax, J.J.; Borger, M.A.; Brotons, C.; Chew, D.P.; et al. 2015 ESC Guidelines for the management of acute coronary syndromes in patients presenting without persistent ST-segment elevation: Task Force for the Management of Acute Coronary Syndromes in Patients Presenting without Persistent ST-Segment Elevation of the European Society of Cardiology (ESC). Eur. Heart J. 2016, 37, 267-315. [PubMed]

3. Eitel, I.; de Waha, S.; Wöhrle, J.; Fuernau, G.; Lurz, P.; Pauschinger, M.; Desch, S.; Schuler, G.; Thiele, H. Comprehensive prognosis assessment by CMR imaging after ST-segment elevation myocardial infarction. J. Am. Coll. Cardiol. 2014, 64, 1217-1226. [CrossRef] [PubMed]

4. White, H.D.; Norris, R.M.; Brown, M.A.; Brandt, P.W.; Whitlock, R.M.; Wild, C.J. Left ventricular end-systolic volume as the major determinant of survival after recovery from myocardial infarction. Circulation 1987, 76 , 44-51. [CrossRef] [PubMed]

5. $\quad$ Eitel, I.; Stiermaier, T.; Lange, T.; Rommel, K.-P.; Koschalka, A.; Kowallick, J.T.; Lotz, J.; Kutty, S.; Gutberlet, M.; Hasenfuß, G.; et al. Cardiac Magnetic Resonance Myocardial Feature Tracking for Optimized Prediction of Cardiovascular Events Following Myocardial Infarction. JACC Cardiovasc. Imaging 2018, 11, 1433-1444. [CrossRef] [PubMed]

6. Mangion, K.; Carrick, D.; Carberry, J.; Mahrous, A.; McComb, C.; Oldroyd, K.G.; Eteiba, H.; Lindsay, M.; McEntegart, M.; Hood, S.; et al. Circumferential Strain Predicts Major Adverse Cardiovascular Events Following an Acute ST-Segment-Elevation Myocardial Infarction. Radiology 2019, 290, 329-337. [CrossRef] 
7. Santos-Gallego, C.G.; Requena-Ibanez, J.A.; San Antonio, R.; Ishikawa, K.; Watanabe, S.; Picatoste, B.; Flores, E.; Garcia-Ropero, A.; Sanz, J.; Hajjar, R.J.; et al. Empagliflozin Ameliorates Adverse Left Ventricular Remodeling in Nondiabetic Heart Failure by Enhancing Myocardial Energetics. J. Am. Coll. Cardiol. 2019, 73, 1931-1944. [CrossRef]

8. Santos-Gallego, C.G.; Vahl, T.P.; Goliasch, G.; Picatoste, B.; Arias, T.; Ishikawa, K.; Njerve, I.U.; Sanz, J.; Narula, J.; Sengupta, P.P.; et al. Sphingosine-1-Phosphate Receptor Agonist Fingolimod Increases Myocardial Salvage and Decreases Adverse Postinfarction Left Ventricular Remodeling in a Porcine Model of Ischemia/Reperfusion. Circulation 2016, 133, 954-966. [CrossRef]

9. Hoit, B.D. Left atrial size and function: Role in prognosis. J. Am. Coll. Cardiol. 2014, 63, 493-505. [CrossRef]

10. Moller, J.E.; Hillis, G.S.; Oh, J.K.; Seward, J.B.; Reeder, G.S.; Wright, R.S.; Park, S.W.; Bailey, K.R.; Pellikka, P.A. Left atrial volume: A powerful predictor of survival after acute myocardial infarction. Circulation 2003, 107, 2207-2212. [CrossRef]

11. Schuster, A.; Backhaus, S.J.; Stiermaier, T.; Navarra, J.-L.; Uhlig, J.; Rommel, K.-P.; Koschalka, A.; Kowallick, J.T.; Lotz, J.; Gutberlet, M.; et al. Left Atrial Function with MRI Enables Prediction of Cardiovascular Events after Myocardial Infarction: Insights from the AIDA STEMI and TATORT NSTEMI Trials. Radiology 2019, 293, 292-302. [CrossRef] [PubMed]

12. Fang, F.; Lee, A.P.-W.; Yu, C.-M. Left atrial function in heart failure with impaired and preserved ejection fraction. Curr. Opin. Cardiol. 2014, 29, 430-436. [CrossRef] [PubMed]

13. Pennell, D.J. Cardiovascular magnetic resonance. Circulation 2010, 121, 692-705. [CrossRef] [PubMed]

14. Schuster, A.; Morton, G.; Chiribiri, A.; Perera, D.; Vanoverschelde, J.-L.; Nagel, E. Imaging in the management of ischemic cardiomyopathy: Special focus on magnetic resonance. J. Am. Coll. Cardiol. 2012, 59, 359-370. [CrossRef]

15. Tadic, M. The right atrium, a forgotten cardiac chamber: An updated review of multimodality imaging. J. Clin. Ultrasound 2015, 43, 335-345. [CrossRef]

16. Kowallick, J.T.; Kutty, S.; Edelmann, F.; Chiribiri, A.; Villa, A.; Steinmetz, M.; Sohns, J.M.; Staab, W.; Bettencourt, N.; Unterberg-Buchwald, C.; et al. Quantification of left atrial strain and strain rate using Cardiovascular Magnetic Resonance myocardial feature tracking: A feasibility study. J. Cardiovasc. Magn. Reson. 2014, 16, 60. [CrossRef]

17. Kowallick, J.T.; Morton, G.; Lamata, P.; Jogiya, R.; Kutty, S.; Hasenfuß, G.; Lotz, J.; Nagel, E.; Chiribiri, A.; Schuster, A. Quantification of atrial dynamics using cardiovascular magnetic resonance: Inter-study reproducibility. J. Cardiovasc. Magn. Reson. 2015, 17, 36. [CrossRef]

18. Shinomiya, H.; Fukuda, N.; Takeichi, N.; Soeki, T.; Shinohara, H.; Yui, Y.; Tamura, Y.; Oki, T. Echocardiographic Assessment of Right Atrial Function in Patients with Myocardial Infarction with Reference to Obstructive Lesions of the Coronary Arteries. Jpn. Circ. J. 1998, 62, 393-398. [CrossRef]

19. Quraini, D.; Pandian, N.G.; Patel, A.R. Three-dimensional echocardiographic analysis of right atrial volume in normal and abnormal hearts: Comparison of biplane and multiplane methods. Echocardiography 2012, 29, 608-613. [CrossRef]

20. Ersbøll, M.; Valeur, N.; Mogensen, U.M.; Andersen, M.J.; Møller, J.E.; Velazquez, E.J.; Hassager, C.; Søgaard, P.; Køber, L. Prediction of all-cause mortality and heart failure admissions from global left ventricular longitudinal strain in patients with acute myocardial infarction and preserved left ventricular ejection fraction. J. Am. Coll. Cardiol. 2013, 61, 2365-2373. [CrossRef]

21. Ersbøll, M.; Andersen, M.J.; Valeur, N.; Mogensen, U.M.; Waziri, H.; Møller, J.E.; Hassager, C.; Søgaard, P.; Køber, L. The prognostic value of left atrial peak reservoir strain in acute myocardial infarction is dependent on left ventricular longitudinal function and left atrial size. Circ. Cardiovasc. Imaging 2013, 6, 26-33. [CrossRef] [PubMed]

22. Saha, S.K.; Söderberg, S.; Lindqvist, P. Association of Right Atrial Mechanics with Hemodynamics and Physical Capacity in Patients with Idiopathic Pulmonary Arterial Hypertension: Insight from a Single-Center Cohort in Northern Sweden. Echocardiography 2016, 33, 46-56. [CrossRef] [PubMed]

23. Wright, L.M.; Dwyer, N.; Wahi, S.; Marwick, T.H. Association with right atrial strain with right atrial pressure: An invasive validation study. Int. J. Cardiovasc. Imaging 2018, 34, 1541-1548. [CrossRef] [PubMed]

24. Yan, P.; Sun, B.; Shi, H.; Zhu, W.; Zhou, Q.; Jiang, Y.; Zhu, H.; Huang, G. Left atrial and right atrial deformation in patients with coronary artery disease: A velocity vector imaging-based study. PLoS ONE 2012, 7, e51204. [CrossRef] 
25. Mantziari, L.; Kamperidis, V.; Ventoulis, I.; Damvopoulou, E.; Giannakoulas, G.; Efthimiadis, G.; Paraskevaidis, S.; Vassilikos, V.; Ziakasm, A.; Karvounism, H.; et al. Increased Right Atrial Volume Index Predicts Low Duke Activity Status Index in Patients with Chronic Heart Failure. Hell. J. Cardiol. 2013, $54,32-38$.

26. Thiele, H.; Wöhrle, J.; Hambrecht, R.; Rittger, H.; Birkemeyer, R.; Lauer, B.; Neuhaus, P.; Brosteanu, O.; Sick, P.; Wiemer, M.; et al. Intracoronary versus intravenous bolus abciximab during primary percutaneous coronary intervention in patients with acute ST-elevation myocardial infarction: A randomised trial. Lancet 2012, 379, 923-931. [CrossRef]

27. Thiele, H.; de Waha, S.; Zeymer, U.; Desch, S.; Scheller, B.; Lauer, B.; Geisler, T.; Gawaz, M.; Gunkel, O.; Bruch, L.; et al. Effect of aspiration thrombectomy on microvascular obstruction in NSTEMI patients: The TATORT-NSTEMI trial. J. Am. Coll. Cardiol. 2014, 64, 1117-1124. [CrossRef]

28. Eitel, I.; Wöhrle, J.; Suenkel, H.; Meissner, J.; Kerber, S.; Lauer, B.; Pauschinger, M.; Birkemeyer, R.; Axthelm, C.; Zimmermann, R.; et al. Intracoronary compared with intravenous bolus abciximab application during primary percutaneous coronary intervention in ST-segment elevation myocardial infarction: Cardiac magnetic resonance substudy of the AIDA STEMI trial. J. Am. Coll. Cardiol. 2013, 61, 1447-1454. [CrossRef]

29. Kowallick, J.T.; Morton, G.; Lamata, P.; Jogiya, R.; Kutty, S.; Lotz, J.; Hasenfuß, G.; Nagel, E.; Chiribiri, A.; Schuster, A. Inter-study reproducibility of left ventricular torsion and torsion rate quantification using MR myocardial feature tracking. J. Magn. Reson. Imaging 2016, 43, 128-137. [CrossRef]

30. Morton, G.; Schuster, A.; Jogiya, R.; Kutty, S.; Beerbaum, P.; Nagel, E. Inter-study reproducibility of cardiovascular magnetic resonance myocardial feature tracking. J. Cardiovasc. Magn. Reson. 2012, 14, 43. [CrossRef]

31. Schuster, A.; Stahnke, V.-C.; Unterberg-Buchwald, C.; Kowallick, J.T.; Lamata, P.; Steinmetz, M.; Kutty, S.; Fasshauer, M.; Staab, W.; Sohns, J.M.; et al. Cardiovascular magnetic resonance feature-tracking assessment of myocardial mechanics: Intervendor agreement and considerations regarding reproducibility. Clin. Radiol. 2015, 70, 989-998. [CrossRef] [PubMed]

32. Kowallick, J.T.; Silva Vieira, M.; Kutty, S.; Lotz, J.; Hasenfu, G.; Chiribiri, A.; Schuster, A. Left Atrial Performance in the Course of Hypertrophic Cardiomyopathy: Relation to Left Ventricular Hypertrophy and Fibrosis. Investig. Radiol. 2017, 52, 177-185. [CrossRef] [PubMed]

33. de Waha, S.; Eitel, I.; Desch, S.; Scheller, B.; Böhm, M.; Lauer, B.; Gawaz, M.; Geisler, T.; Gunkel, O.; Bruch, L.; et al. Thrombus Aspiration in ThrOmbus containing culpRiT lesions in Non-ST-Elevation Myocardial Infarction (TATORT-NSTEMI): Study protocol for a randomized controlled trial. Trials 2013, 14, 110. [CrossRef] [PubMed]

34. Martin Bland, J.; Altman, D. Statistical methods for assessing agreement between two methods of clinical measurement. Lancet 1986, 327, 307-310. [CrossRef]

35. Willens, H.J.; Fertel, D.P.; Qin, J.; Labrador, E.; Lowery, M.H. Effects of age and pulmonary arterial hypertension on the different phases of right atrial function. Int. J. Cardiovasc. Imaging 2008, 24, 703-710. [CrossRef]

36. Wang, Y.; Zhang, Y.; Ma, C.; Guan, Z.; Liu, S.; Zhang, W.; Li, Y.; Yang, J. Evaluation of Left and Right Atrial Function in Patients with Coronary Slow-Flow Phenomenon Using Two-Dimensional Speckle Tracking Echocardiography. Echocardiography 2016, 33, 871-880. [CrossRef]

37. Tadic, M.; Cuspidi, C.; Kocijancic, V.; Celic, V.; Vukomanovic, V. Does Left Ventricular Geometric Patterns Impact Right Atrial Phasic Function? Findings from the Hypertensive Population. Echocardiography 2016, 33 , 1186-1194. [CrossRef]

38. Von Roeder, M.; Kowallick, J.T.; Rommel, K.-P.; Blazek, S.; Besler, C.; Fengler, K.; Lotz, J.; Hasenfuß, G.; Lücke, C.; Gutberlet, M.; et al. Right atrial-right ventricular coupling in heart failure with preserved ejection fraction. Clin. Res. Cardiol. 2020, 109, 54-66. [CrossRef]

39. Stillman, A.E.; Oudkerk, M.; Bluemke, D.A.; de Boer, M.J.; Bremerich, J.; Garcia, E.V.; Gutberlet, M.; van der Harst, P.; Hundley, W.G.; Jerosch-Herold, M.; et al. Imaging the myocardial ischemic cascade. Int. J. Cardiovasc. Imaging 2018, 34, 1249-1263. [CrossRef]

40. Posina, K.; McLaughlin, J.; Rhee, P.; Li, L.; Cheng, J.; Schapiro, W.; Gulotta, R.J.; Berke, A.D.; Petrossian, G.A.; Reichek, N.; et al. Relationship of phasic left atrial volume and emptying function to left ventricular filling pressure: A cardiovascular magnetic resonance study. J. Cardiovasc. Magn. Reson. 2013, 15, 99. [CrossRef] 
41. von Roeder, M.; Rommel, K.-P.; Kowallick, J.T.; Blazek, S.; Besler, C.; Fengler, K.; Lotz, J.; Hasenfuß, G.; Lücke, C.; Gutberlet, M.; et al. Influence of Left Atrial Function on Exercise Capacity and Left Ventricular Function in Patients with Heart Failure and Preserved Ejection Fraction. Circ. Cardiovasc. Imaging 2017, 10, e005467. [CrossRef] [PubMed]

42. Govindan, M.; Kiotsekoglou, A.; Saha, S.K.; Camm, A.J. Right atrial myocardial deformation by two-dimensional speckle tracking echocardiography predicts recurrence in paroxysmal atrial fibrillation. J. Echocardiogr. 2017, 15, 166-175. [CrossRef] [PubMed]

43. Sicari, R. Right atrial function: A blind spot in a blind spot. Int. J. Cardiol. 2018, 255, 212. [CrossRef] [PubMed]

44. Liu, B.; Dardeer, A.M.; Moody, W.E.; Edwards, N.C.; Hudsmith, L.E.; Steeds, R.P. Normal values for myocardial deformation within the right heart measured by feature-tracking cardiovascular magnetic resonance imaging. Int. J. Cardiol. 2018, 252, 220-223. [CrossRef]

45. Backhaus, S.J.; Kowallick, J.T.; Stiermaier, T.; Lange, T.; Koschalka, A.; Navarra, J.-L.; Lotz, J.; Kutty, S.; Bigalke, B.; Gutberlet, M.; et al. Culprit vessel-related myocardial mechanics and prognostic implications following acute myocardial infarction. Clin. Res. Cardiol. 2019, 37, 267. [CrossRef]

46. Goldstein, J.A. Acute right ventricular infarction: Insights for the interventional era. Curr. Probl. Cardiol. 2012, 37, 533-557. [CrossRef]

47. Padeletti, M.; Cameli, M.; Lisi, M.; Zacà, V.; Tsioulpas, C.; Bernazzali, S.; Maccherini, M.; Mondillo, S. Right atrial speckle tracking analysis as a novel noninvasive method for pulmonary hemodynamics assessment in patients with chronic systolic heart failure. Echocardiography 2011, 28, 658-664. [CrossRef]

(C) 2020 by the authors. Licensee MDPI, Basel, Switzerland. This article is an open access article distributed under the terms and conditions of the Creative Commons Attribution (CC BY) license (http://creativecommons.org/licenses/by/4.0/). 